\title{
Le Portugal, un refuge aux deux facettes
}

Résumé:

N'étant pas directement intervenu dans les conflits de la Seconde Guerre mondiale, en sa qualité de pays "neutre", le Portugal a pourtant été au cœur de représentations et aspirations diverses et contradictoires. Terre d'accueil ou de passage, le pays restait aux mains d'un pouvoir totalitaire castrateur et menaçant, tout en étant vu par les réfugiés comme une porte ouverte sur la liberté. Au-delà des éléments traditionnellement associés au Portugal de l'époque, le roman d'Helena Marques (O Bazar alemão, 2010) et le documentaire de Nicholas Oulman (Debaixo do céu, 2018, 75') dévoilent un Portugal méconnu à travers les voix de ceux et celles qui en ont été les témoins. Ces récits d'existence participent ainsi au savoir dynamique construit sur cette période, à partir d'archives toujours renouvelées.

Mots-clés:

Portugal, Seconde Guerre mondiale, salazarisme, archives

Abstract:

Not having directly intervened in the conflicts of the Second World War, as a "neutral" country, Portugal was nevertheless at the heart of diverse and contradictory representations and hopes. A welcoming land or a passageway, the country remained in the hands of a castrating and threatening totalitarian power, while being seen by refugees as an open door to freedom. Beyond the traditionally associated elements of Portugal at the time, Helena Marques' novel (O Bazar alemão, 2010) and Nicholas Oulman's documentary (Debaixo do céu, 2018, 75') reveal a little-known Portugal through the voices of those who witnessed it. Thus, these stories of existence participate in the dynamic knowledge built upon this period, from constantly renewed archives

Keywords:

Portugal, Second World War, salazarism, archives 
[...] La côte du Portugal demeurait le dernier refuge des émigrants pour qui la justice, la liberté et la tolérance étaient plus précieuses que le sol natal et que la certitude du pain quotidien. Celui qui ne pouvait partir pour atteindre à la terre promise, l'Amérique, était perdu [...] L'homme par lui-même n'était plus rien ; un passeport valable était tout.

Erich Maria Remarque, La Nuit de Lisbonne: 8

Dans la biographie qu'il consacre à la diva du fado Amália Rodrigues (1920-1999), le journaliste Miguel Carvalho dévoile le visage complexe et méconnu de celle qu'on a souvent accusée d'être complice du régime dictatorial. Parfaitement consciente de son rang, du milieu modeste dont elle est issue, et d'une grande lucidité sur ce qui a construit sa carrière, elle a toujours revendiqué son entière liberté et son refus de se voir instrumentalisée par le pouvoir politique. Elle avoue:

Durante a guerra, eu sabia que havia um homem chamado Hitler a matar as pessoas. Mas era tão longe, e eu andava tão encantada com o meu sucesso enquanto rapariga - não era nada feia - e como artista. As boîtes estavam cheias de refugiados, gente bonita e bem vestida. Era um mundo que eu estava a descobrir.

[Pendant la guerre, je savais qu'il y avait un homme appelé Hitler qui tuait des gens. Mais c'était si loin, et j'étais si enchantée de mon succès en tant que jeune femme - j'étais plutôt jolie - et en tant qu'artiste. Les boîtes de nuit étaient emplies de réfugiés, des gens beaux et bien habillés. C'était tout un monde que j'étais en train de découvrir] (Carvalho 2020 :48)

Amália a été le témoin de ce Portugal des années 1940 aux deux visages, où les exilés et réfugiés concourent au cosmopolitisme des mœurs d'un pays qui offre l'hospitalité, mais qui reste indifférent à la souffrance de ces êtres humains en fuite, tout autant qu'à celle de la majorité de la population portugaise de l'époque. Alors qu'Hitler a pris le pouvoir et que les lois raciales de Nuremberg ont été proclamées en Allemagne, la dictature s'est durcie au Portugal depuis les années 1930. N'étant pas directement intervenu dans les conflits de la Seconde Guerre mondiale, en sa qualité de pays "neutre", le Portugal a pourtant été au cœur de représentations et aspirations diverses et contradictoires. Terre d'accueil ou de passage, le pays restait aux mains d'un pouvoir totalitaire castrateur et menaçant, qui se livrait par ailleurs à des activités économiques lucratives souvent peu avouables. Mais dans les yeux des indésirables réfugiés condamnés à la fuite, le Portugal apparaît comme une porte ouverte sur la liberté outre-Atlantique.

Le roman O Bazar alemão et le documentaire Debaixo do Céu nous offrent deux manières de donner corps à ce pays qui exerce sur les réfugiés une telle force d'attraction, symbole d'ultime espoir. Ces deux œuvres trouvent leur source dans le même geste: puiser dans des archives méconnues donnant la parole aux témoins d'événements que tous connaissent peu ou prou, 
mais sur lesquels elles transmettent un regard singulier, d'autant plus singulier qu'elles touchent le lecteur et le spectateur par leur universalité. Selon Maria de Fátima Marinho, la stratégie de Helena Marques, à travers "a veracidade do que vai narrar, valendo-se de documentos encontrados, escudando-se embora num anonimato que os nomes supostos das personagens proporcionam" [ "la véracité de ce qu'elle va raconter, en se prévalant de documents trouvés, tout en se retranchant cependant derrière un anonymat permis par les noms fictifs des personnages"], provoque "[o] envolvimento emocional do leitor, que se sente atingido por relatos apresentados como verídicos" [ "l'implication émotionnelle du lecteur, qui se sent touché par ces récits présentés comme véridiques"] (Marinho 2011 : 237). C'est cette même émotion qui happe également le spectateur de Debaixo do céu, qui devient le témoin d'expériences intimes jamais dévoilées. Sans pointer explicitement la dictature de Salazar, ces voix, témoins directs ou personnages de fiction, rendent compte d'un décentrement: dans le roman, celui-ci est lié à la position géographique de Madère, éloignée de la métropole, mais sur laquelle plane le fantôme du régime totalitaire; dans le documentaire, il est lié à la mémoire que les témoins convoquent - celle des enfants qu'ils étaient lorsqu'ils ont dû fuir leur pays, à un âge où ils n'avaient pas encore conscience de l'ampleur des crimes nazis et de leurs conséquences. Ce décentrement temporel et géographique se double de la volonté de donner la parole à des témoins jusque-là non entendus, et dont il faut réinventer en partie ce qu'ils ont à nous dire. Helena Marques et Nicholas Oulman donnent ainsi corps à ce que Éric Ketelaar appelle la "narration tacite des archives", la nécessité de réinterpréter celles-ci dans un processus dynamique, au gré de l'époque à laquelle le document est lu (Ketelaar 2006 :69). Georges Didi-Huberman évoque, quant à lui, l'importance du montage des archives et des images, en tant qu'il permet de penser et de montrer (Didi-Huberman 2003: 172), dans la mesure où les archives sans montage ne constituent jamais la vérité pure, absolue, unique (idem: 166). L'archive est donc toujours en construction car

chaque découverte y surgit comme une brèche dans l'histoire conçue, une singularité provisoirement inqualifiable que le chercheur va tenter de raccommoder au tissu de tout ce qu'il sait déjà pour produire, si possible, une histoire repensée de l'événement en question [...] Elle n'est pas pour autant le pur et simple "reflet" de l'événement, ni sa pure et simple "preuve". Car elle est toujours à élaborer par recoupements incessants, par montage avec d'autres archives (idem: 126-127). ${ }^{2}$

Par le montage des images et des mots, par la fiction et le croisement de sources historiques, publiques ou privées, collectives ou plus intimes, Helena Marques et Nicholas Oulman ouvrent cette brèche, repensent les événements et enrichissent le caléidoscope des récits d'existence liés à la Seconde Guerre mondiale, et ayant le Portugal pour toile de fond. 
Cadernos de Literatura Comparada

Le Portugal, un refuge aux deux facettes

\section{Debaixo do céu : le temps retrouvé d'un ciel limpide}

Né au sein d'une famille juive française installée au Portugal depuis 1920, et fils d'Alain Oulman (1928-1990) - connu surtout pour avoir collaboré avec Amália Rodrigues dont il a composé de nombreuses chansons -, Nicholas Oulman fait dialoguer dans ce documentaire des archives d'époque puisées notamment dans les fonds de la ville de Lisbonne ou du musée de l'Holocauste de Washington (cf. crédits indiqués sur le générique de fin) et la voix de sept témoins, que d'aucuns appelleraient sans doute des "survivants". ${ }^{3}$ Les images de Berlin en ruines ouvrent et clôturent le film. Les premières sont en noir et blanc, celles de la fin légèrement colorées, associées à l'affirmation du message d'espoir en l'homme transmis par Lolita Goldstein dans les derniers mots du film - tout homme a le pouvoir d'influencer les choses à son échelle, même dans les plus petits gestes quotidiens, nous dit-elle. Entre ces deux moments, des images d'archives en noir et blanc, fixes ou animées, nous font voyager de l'Allemagne vers le Portugal, but commun de toutes les personnes qui s'expriment ici, sans que l'on ne voie jamais leur visage; chacun de ces visages de témoins, accompagné du nom de la personne à laquelle il correspond, et du titre des séquences que chacun d'eux commente, est livré sur le générique de fin. C'est là l'une des caractéristiques du point de vue adopté par ce film: des enfants qui ont dû, du jour au lendemain, quitter ce qu'ils possédaient, ce qu'ils étaient, passer au statut de réfugiés, et qui se remémorent, des décennies plus tard, leur regard de l'époque sur une situation que la plupart ne comprenait pas encore. L'un des exemples les plus éloquents est le récit du bombardement du train où circule, alors âgé d'environ 4 ans, Fred Manasse. À l'époque, il prend ce bombardement pour un spectacle de magie et un feu d'artifice - il ne saura la vérité que soixante-dix ans plus tard. Il s'agit donc de récits intimistes, d'expériences personnelles, de détails parfois anecdotiques, mais qui trouvent leur place dans l'Histoire, de manière à montrer à quel point le Portugal fut un pays important dans l'aide aux réfugiés (Sobral 2019). Olivier Ypsilantis commente la manière dont Debaixo do Céu déroge à la fois au style classique du documentaire, et aux images du Portugal traditionnellement associées à la Seconde Guerre mondiale:

Ce film évite volontairement les images de guerre style documentaire. Il se concentre sur des images plutôt intimistes, des enfants à l'école, en famille, des images de fuite aussi, avec notamment le passage (risqué) des Pyrénées. Car la France qui est relativement sûre pour ces Juifs d'Europe centrale devient vite menaçante avec l'invasion allemande, invasion en deux temps avec cette zone dite "libre", jusqu'en novembre 1942. Ce mouvement de fuite vers le Portugal passe par l'Espagne, une Espagne presqu'entièrement dévastée par la Guerre Civile de 19361939 [...] Nicholas Oulman est entré en contact avec ces femmes et ces hommes qui témoignent devant sa caméra par l'intermédiaire de Carlos Guerreiro qui gère un blog, "Aterrem em Portugal!", blog qui se propose de rassembler des documents et des témoignages sur le Portugal de la période 1939-1945 [...] La mémoire des enfants ne s'intéresse pas au régime de Salazar auquel il n'est pas fait allusion, sauf une fois, en passant, par Sylvain, lorsqu'il évoque son séjour à Figueira da Foz, avec ce Portugais qui ne rêve que de quitter le Portugal et ce régime qu'il exècre. 
Pas la moindre allusion à Aristides de Sousa Mendes car, une fois encore, Nicholas Oulman a pris le parti (défendable) de ne pas évoquer ce qui l'est presque toujours (et peut-être même toujours) dans un film à caractère documentaire relatif aux Juifs et au Portugal d'alors. Debaixo do Céu est un film en marge du documentaire au sens courant du genre. On devine par ailleurs sans peine le travail de montage, considérable et essentiel, à partir de nombreuses heures d'entrevues certaines d'entre elles ont duré trois heures alors que le film dure à peine plus d'une heure et quart (Ypsilantis 2019).

Le parti-pris du film repose donc sur la volonté de montrer un Portugal méconnu, comme si le réalisateur voulait surprendre le spectateur tout autant que l'enfant fugitif a été surpris, au gré des espaces traversés et des personnes rencontrées. Le réalisateur se souvient d'ailleurs comment, lorsqu'il était enfant, ses parents accueillaient des réfugiés. C'est donc aussi une partie de son histoire qu'il nous raconte, ou d'une histoire qui aurait pu être un peu la sienne, et dans laquelle se reflète une partie de l'histoire de l'humanité : cette ville de Berlin détruite, cela peut aussi être Alep, nous dit-il.4

Le film obéit à une double construction: d'un côté, les images d'archives se succèdent, comme divers tableaux illustrant le contexte de l'époque et les différentes étapes de la fuite des réfugiés ; de l'autre, le réalisateur fait correspondre ces images avec le récit, en voix off, de témoins qui nous racontent des épisodes de leur fuite. Il y a plus d'une vingtaine de séquences au cours desquelles une voix différente s'exprime. Nous comprenons vite au fur et à mesure qu'il s'agit des mêmes voix alternées au gré de ce que nous pouvons identifier comme étant les trois parties du film: les circonstances du départ, puis le voyage et la fuite, puis enfin l'arrivée au Portugal. Le rythme des images, passant comme au ralenti, et le fait de ne pas voir qui parle, nous fait traverser, à nous spectateurs, immergés, l'expérience de cette errance. Ce n'est qu'à la minute 48 que le ciel s'ouvre enfin, avec la traversée de la frontière qui sépare l'Espagne et le Portugal. Certains arrivent à Lisbonne, d'autres à Figueira da Foz. Tous sont unanimes: ce qui les frappe d'abord, c'est le soleil, les paysages verts, les maisons bien tenues, un pays qui leur semble libre bien qu'humble; puis les gens, la nourriture. Henny Porter insiste sur le fait qu'au Portugal tout était authentique (elle utilise les mots "real", "a sério", qui s'opposent à l'économie de la guerre, à l' "Ersatz" allemand): c'est clairement pour elle un renouveau, le début d'une nouvelle vie. Deux autres témoins insistent par ailleurs sur ce Portugal "empli de réfugiés", aidés par des organisations juives et des organisations internationales (l'une des témoins raconte comment elle a été engagée comme interprète pour accueillir et aider les réfugiés débarquant dans les gares). Le régime de Salazar avait créé des camps pour ces réfugiés, souhaitant les parquer à part, loin de la capitale, afin qu'ils ne viennent pas perturber la stabilité que le régime parvenait à maintenir; ils n'étaient pas censés pouvoir quitter ces villes sans autorisation (Meneses, 2015: 264). N. Oulman insiste sur le fait que ces réfugiés étaient bien souvent plus cultivés et de pays plus "modernes" à bien des égards, par rapport à la société portugaise dans laquelle ils arrivaient, et que cette prétendue restriction de circulation n'a pas toujours véritablement fonctionné (Sobral 2019). Sylvain Bromberger raconte même comment 
il a pu faire du tourisme au Portugal pendant deux jours, grâce à une Portugaise qui l'a emmené visiter Tomar, Batalha et Coimbra.

Aucun détail n'est donné au cours du film sur la provenance de ces archives. Les images sont illustratives, dans la mesure où elles viennent imprimer des visages, des corps, des paysages, des objets existants sur des récits qui en étaient jusque-là dépourvus (ces jeunes enfants sont partis sans rien, ou presque, et n'ont donc pas pu constituer d'archives personnelles sur cette période), mais elles sont surtout suggestives, car ces voix qui s'expriment en tant que témoins d'une grande aventure humaine et collective, viennent mettre des mots sur ces images, et sans doute sur d'autres que chacun et chacune d'entre nous a pu voir sur cette période. Les images s'enchaînent au fil de l'évocation par les témoins de la tension croissante en Allemagne, des déportations des juifs, de la décision du départ, souvent à l'occasion d'une opportunité saisie (trois places restantes dans le wagon d'un train partant pour la France, par exemple), mais aussi de la traversée des Pyrénées, d'une Espagne dévastée par la guerre civile où les enfants viennent mendier du pain sur les rails en échange de l'eau du puits ... Parfois, voix et images coïncident: au récit de l'arrivée à la gare d'Irun, correspondent les images d'une foule s'entassant sur les quais; au récit sur la manière dont deux enfants parviennent à se procurer des passeports à Bayonne, correspondent les images de files d'attente devant le consulat; lorsque nous est raconté l'épisode du bombardement pris pour un feu d'artifice, que le petit garçon voit à travers un petit trou du wagon, nous voyons un bombardement à travers une fente ; lorsqu'une témoin raconte comment du jour au lendemain, l'accès à l'école lui a été interdit, nous voyons à l'écran des images d'élèves heureux dans la classe ou en récréation - images de ce qui a été, pour cette petite fille d'alors, irrémédiablement perdu. Le seul exemple flagrant d'une correspondance totale entre le récit et l'archive personnelle consiste sans doute dans la photographie d'un chœur d'enfants sur une scène, accompagnés de musiciens: Eva Arond raconte comment, en 1941, alors que les théâtres sont interdits aux juifs, son école organise un concert dans un théâtre appartenant à la communauté hébraïque de Hambourg, et fait jouer des musiciens professionnels qui avaient perdu leur travail. Elle dit être la seule au monde à avoir gardé une photographie de cet événement. Hors du temps et de l'espace, la captation de ce moment clandestin prend vie publiquement par ce récit.

Mais le parallèle entre ce qui est montré et ce qui est dit crée également des résonnances, des rapprochements, sans qu'il s'agisse d'une superposition exacte entre les images et les mots. Lorsque Lolita Goldstein s'exprime pour raconter les circonstances dans lesquelles, le 1er mai 1933, elle décide de partir pour le Portugal, d'où son père l'avait avertie qu'il fallait fuir l'Allemagne (en ouvrant le journal, elle lit le gros titre "Les étudiants juifs ne peuvent participer à la parade"), défilent à l'écran des images de Berlin en ruines. Au fur et à mesure que se précisent les sentiments de l'adolescente à l'époque, les "frissons" que lui procure ce souvenir (le mot est évoqué à deux reprises dans le récit de cet épisode), les images zooment sur tel ou tel édifice de la ville. Face à cette atmosphère de destruction, le sentiment de rejet exprimé par Lolita Goldstein gagne ainsi davantage d'épaisseur. C'est là qu'apparaît tout le secret du montage, ce qu'on peut associer à ce que Georges Didi-Huberman appelle le travail 
d'imagination et de spéculation nécessaire au montage des archives: "L'image d'archive n'est qu'un objet entre mes mains, un tirage photographique indéchiffrable et insignifiant tant que je n'ai pas établi la relation - imaginative et spéculative - entre ce que je vois ici et ce que je sais par ailleurs" (Didi-Huberman 2003: 142), l'imagination étant le "temps de travail des images" (idem: 149). Nicholas Oulman dit avoir recherché ce décalage, son objectif étant de faire correspondre le montage des images avec un montage sonore capable de nous révéler celles-ci autrement, de nous raconter des détails a priori invisibles à l'image (Sobral 2019), mais auxquels la voix donne une existence, une épaisseur sensible. Ces témoins décrivent ce sentiment d'être en fuite, même quand les enfants qu'ils étaient n'avaient reçu aucune explication sur les raisons de leur départ; une autre insiste sur le fait que l'essentiel était de tout faire pour rester "invisible", car elle "savait" ce qu'il se passait avec les juifs, les nouvelles des déportations étant connues bien que taboues dans les familles. Et quand une autre dit que les choses "avaient empiré en Allemagne", surgissent à l'écran les images des autodafés réalisés par les nazis en pleine rue, avec des gros plans sur les flammes. Le film est donc "à côté" de ce qu'on pourrait parfois en attendre et fait écho à la surprise du réalisateur lui-même:

os relatos que me foram contados não eram exatamente aquilo de que eu estava à espera. Eu estava à espera de mais dramatismo, de mais intensidade dramática nos relatos e de uma coisa mais de adulto. Deparei-me com memórias de crianças. Apercebi-me rapidamente de que havia ali qualquer coisa que era muito inocente mas ao mesmo tempo forte, recorda.

[les récits qui m'ont été livrés n'étaient pas exactement tels que je les imaginais. J'attendais plus de dramatisme, plus d'intensité dramatique dans les récits, des choses plus liées au monde adulte. J'ai été confronté à des souvenirs d'enfants. Je me suis vite rendu compte qu'il y avait là quelque chose de très innocent mais en même temps de fort, se rappelle-t-il]. (Pereira 2019)

Ce qui naît de cet entre-deux, de cette rencontre entre l'attente et la surprise, crée tout l'intérêt de ce montage. Ces récits de témoins dépourvus d'archives personnelles viennent faire parler des images d'archives existantes, à travers l'expression de leurs émotions intimes qui affleurent souvent dans la voix, comme dans ce récit ému où un témoin avoue n'avoir jamais cessé de rechercher sa sœur, dont il ignore ce qu'elle est devenue après qu'elle a été envoyée dans un camp. Ils insèrent ainsi leur histoire dans l'Histoire, le montage révélant les archives tout autant que les archives révèlent le montage.

À la minute 52, Sylvain Bromberger, devenu citoyen américain à 18 ans, engagé dans l'armée américaine et revenu en Europe, raconte qu'il donne des cours de français à un bibliothécaire de Figueira da Foz car celui-ci voulait être capable de "lui raconter à quel point le régime était horrible"; Fred Manasse, lui, raconte à quel point il ne voulait pour rien au monde quitter le Portugal, tellement il s'y sentait bien, intégré. Paradis pour les uns, enfer pour les autres: ces réfugiés disent trouver un Eldorado et la liberté dans un Portugal où la société en est pourtant privée, le pays étant en proie à un régime dictatorial, répressif et prêt à tout pour maintenir son empire et s'enrichir. Selon Filipe Ribeiro de Meneses, il y a un apparent para- 
Cadernos de Literatura Comparada

Le Portugal, un refuge aux deux facettes

doxe entre le durcissement des règles au Portugal, la fermeture des frontières portugaises, et le nombre croissant de réfugiés qui arrivaient malgré tout et finissaient par dépendre des organisations juives, nationales et internationales:

Dès le déclenchement de la guerre, le Portugal ferma ses frontières à presque tous les réfugiés, mais ceux-ci arrivèrent quand même, ce qui eut un impact significatif sur la vie portugaise pendant le conflit (et donna l'impression durable au pays qu'il avait joué un rôle positif pendant le conflit). Une attitude véritablement humanitaire aurait contribué à sauver de nombreuses autres vies (et pas seulement juives), mais en même temps, les organisations de secours juives furent autorisées à fonctionner dans le pays [...] Mais qu'est-ce qui motiva l'indifférence, voire l'opposition, de Salazar à l'idée que le Portugal joue un rôle majeur dans le sauvetage de vies juives? [...] Au début du conflit, le nombre d'étrangers cherchant refuge au Portugal augmenta encore, et les règlements pour entrer au Portugal se durcirent en conséquence, selon la PVDE, à la fois pour des raisons de sécurité nationale et parce que de nombreux consuls n'avaient pas suivi les instructions récemment envoyées. Leur négligence, ou leur désobéissance, signifiait que les réfugiés juifs qui n'avaient pas le droit de retourner dans leur patrie pénétraient au Portugal, devenant, affirmait-on, un fardeau pour l'État. [...] À la fin de l'année 1940, il y avait encore quelque 10000 réfugiés dans le pays, dont 90\% étaient juifs. Au total, selon Milgram, entre 13500 et 15000 Juifs traversèrent le Portugal pendant la guerre. Hésitant à laisser se développer la concurrence économique, le gouvernement empêcha les réfugiés de travailler, réduisant ceux qui ne pouvaient partir rapidement à un état de dépendance vis-à-vis des organisations caritatives portugaises et étrangères [Meneses 2015: 256-265].

Le double visage du Portugal, dont tous les réfugiés n'avaient pas forcément conscience, est ici bien visible. Non seulement il s'agit d'une dictature qui apparaît comme une possibilité unique de liberté pour les réfugiés, mais il s'agit aussi d'un pays qui a pu accueillir des milliers de réfugiés malgré les mesures prises pour contenir ce flux, pas toujours bien respectées par les consuls, ce qui confère au Portugal de l'époque un paraître tout à fait différent de son être profond. Filipe Ribeiro de Meneses nous rappelle que Salazar agit envers les réfugiés comme il a agi envers son propre peuple, avec indifférence et manque d'empathie, sans compter qu'en 1940, le Portugal prépare les commémorations de l'Exposition du Monde Portugais, destinées à renforcer le sentiment nationaliste et ne souhaite pas voir menacée la stabilité qu'il a réussi à construire (idem: 26). Salazar a donc agi en pensant d'abord à lui, en instrumentalisant la neutralité qu'il avait choisie pour le Portugal - une neutralité bien moins idéologique que politique, pragmatique, "tactique", selon Georges Bensoussan:

Le refus portugais de s'engager dans la guerre aux côtés de l'Axe, par exemple, est à la fois tactique et idéologique. Le régime de Salazar a peu à voir avec le fascisme italien, et moins encore avec le nazisme allemand. Enfermé dans un cléricalisme archaïque, voire quasi paranoïaque, le dictateur catholique voit le Portugal comme un corps malade à purger et à isoler des miasmes 
de l'extérieur. L'afflux de réfugiés fuyant le nazisme puis l'occupation allemande contrarie son programme de mise en quarantaine thérapeutique du Portugal. Entrent toutefois aussi dans cette neutralité des considérations tactiques, celles d'un pays qui n'a plus les moyens de l'empire colonial d'autrefois, un empire de surcroît dispersé que Lisbonne craint de voir basculer vers d'autres souverainetés. La neutralité portugaise est donc aussi celle du réalisme de qui sait ne plus pouvoir compter sur son ancienne grandeur [...] À Lisbonne, Salazar se montre indifférent à la souffrance des réfugiés comme à celle des pauvres de son propre peuple. Informé du génocide en cours, le dictateur catholique ne cherche aucunement à sauver les 4300 "Juifs portugais" des Pays-Bas dont il ne restera qu'un peu moins de 500 survivants en 1945. (Bensoussan 2015: 8-11).

Cette neutralité qui permet d'éclaircir le ciel de fugitifs en quête de leur propre identité, même de manière passagère, de donner un abri à des enfants confrontés au néant dans une ville berlinoise à ciel ouvert, est pourtant la même qui a condamné d'autres Portugais de l'étranger, et qui a noirci toujours un peu plus le ciel des Portugais vivant au Portugal.

\section{O Bazar alemão: l'envers du décor}

Le roman d'Helena Marques nous fait parcourir la vie à Madère entre 1936 et 1939. Alors que l'île est décrite comme une oasis dans laquelle des réfugiés de toutes origines (allemande, hongroise, polonaise) ont trouvé un abri, puis ont décidé de s'installer (Marques 2010: 21), ${ }^{5}$ des Juifs d'origine allemande, vivant pourtant en territoire portugais, sont persécutés par des Allemands nazis souhaitant eux-mêmes appliquer les lois de Nuremberg de 1935 dans des noyaux allemands de l'étranger comme Madère. La délation, des chantages divers, des lettres de dénonciation envoyées à la Gestapo, ont fait partie des moyens utilisés pour persécuter cette population "com imediatas repercussões sobre o Consulado Alemão no Funchal e sobre a vida pessoal e profissional dos cidadãos denunciados" [ "avec d'immédiates répercussions sur le Consulat allemand à Funchal et sur la vie personnelle et professionnelle des citoyens dénoncés"] (idem: 11). Toutes ces personnes inquiétées ont gardé le silence et n'ont jamais fait part de ces discriminations à l'opinion publique, jusqu'à ce qu'en 1999, comme l'explique la note liminaire du roman, soient découvertes des archives dans le Fonds du Consulat allemand de Funchal, qui intègrent à présent les Archives Politiques de la République Fédérale Allemande à Berlin.

À partir de ces archives, la romancière et journaliste Helena Marques, originaire de Madère, a eu pour objectif de recréer l'ambiance de l'époque et ce pan méconnu de l'Histoire: d'un côté, cette persécution absurde, "insólito exercício de um terror doméstico e gratuito" ["insolite exercice d'une terreur domestique et gratuite"] (idem: 13), et de l'autre, les vagues successives de réfugiés arrivant au Portugal et évoquées notamment dans les lettres échangées par les familles entre Madère et Lisbonne:

Cartas de amigos e parentes em Lisboa continuam a informar os madeirenses sobre os constantes fluxos de refugiados da Europa Central, sobretudo judeus, que chegam a Portugal de navio ou 
de comboio, em números cada vez maiores, à procura de abrigo ou, pelo menos, de um patamar de segurança, enquanto aguardam viagem para os Estados Unidos ou para a América do Sul. [des lettres d'amis et de parents proches vivant à Lisbonne continuent à informer les habitants de Madère sur les flux constants de réfugiés venus d'Europe Centrale, surtout des Juifs, qui arrivent au Portugal par bateau ou en train, en nombre toujours plus important, à la recherche d'un abri ou tout au moins d'un certain niveau de sécurité, pendant qu'ils attendent de voyager vers les Etats-Unis ou l'Amérique du Sud]. (Marques 2010:73)

\section{Ou encore :}

Cartas de Lisboa davam-lhe conta da chegada de vagas sucessivas de refugiados, muitos à espera de transporte para os Estados Unidos ou para países da América do Sul, mas outros sem destino programado, trazidos apenas por um urgente sentido de sobrevivência. Parecia já ter começado a debandada, a fuga em massa da Alemanha.

[Des lettres de Lisbonne l'informaient de l'arrivée de vagues successives de réfugiés, beaucoup d'entre eux en attente de transport vers les Etats-Unis ou les pays d'Amérique du Sud, mais d'autres sans destination programmée, amenés seulement par l'urgence d'un instinct de survie. La débandade, la fuite en masse de l'Allemagne, semblait avoir déjà commencé]. (idem: 105)

Madère se trouve donc à la croisée de plusieurs chemins. Spectatrice plus ou moins lointaine de ces flux humains réduits à la fuite et à la recherche de protection, l'île devient le réceptacle d'une partie de cette misère emplie d'espoir. Si le Portugal continental a le plus souvent fait office de pays de transit pour les réfugiés en attente de l'eldorado américain, Madère semble avoir été adoptée par de nombreux réfugiés comme une destination définitive.

Mais le pire guette, puisque ce territoire portugais incarne lui aussi la double facette assumée par le continent, un pays neutre qui, tout en collaborant avec l'ennemi, a accueilli ses réfugiés. L'axe principal du roman se tisse autour du couple Bromberger, des Allemands particulièrement zélés. Non seulement ils s'adonnent tous les mercredis soirs à de petits rituels nazis dans l'un des salons de l'hôtel de la ville (idem: 106), mais ils incarnent la folie de la persécution: ils vont jusqu'à menacer de dénonciation un médecin qui a loué un bureau à un Allemand juif - médecin qui ne se laisse pas intimider, rappelant à ses persécuteurs "que se encontravam em Portugal, onde apenas vigoravam as leis portuguesas, das quais não fazia parte qualquer discriminação contra os Judeus" ["qu'ils se trouvaient au Portugal, où seules les lois portugaises étaient appliquées, dont ne faisait partie aucune discrimination envers les Juifs"] (idem: 29). Les Bromberger exigent également que le couple d'humbles immigrés polonais, Miriam et Izaak Brusov, gérants de la mercerie "O Bazar alemão", arbore dans son espace commercial des drapeaux nazis (idem: 144). Notons l'ironie du nom de la boutique: des Polonais que l'Allemagne a fait fuir trouvent sur l'île portugaise une oasis, où ils reprennent un commerce existant, sans en changer le nom pour ne pas menacer une certaine stabilité; ils sont à nouveau menacés par des procédés absurdes alors qu'ils croyaient avoir trouvé la sérénité, comme si le maintien de ce nom les enfermait dans un cycle de persécutions sans fin. Enfin, 
les Bromberger parviennent à rendre impossible l'union entre Eugen, d'origine allemande, et Elisabeth, une Portugaise née de père allemand et de mère française. L'histoire de cette union empêchée ouvre et ferme le roman : il aura fallu à ces amoureux attendre 3 ans, de 1936 à 1939, pour apprendre que leur mariage est impossible car l'Allemagne refuse de fournir les papiers au consul, sous prétexte qu'un Allemand ne peut épouser une juive, alors qu'Elisabeth est portugaise et ne devrait donc pas être concernée par cette loi. L'intrigue avance au gré de ces étapes qui repoussent sans cesse les limites de l'absurdité, tandis qu'Eugen pressent un enjeu sur lequel il ne peut avoir de prise:

Nem todos disporiam, por certo, como o Dr Franz Schönberg e ele próprio, da segurança de residir e trabalhar na Madeira há já alguns anos, em completa legalidade e integração social - e também invulneráveis, por raciocínio e temperamento, a qualquer processo intimidatório e, muito menos ainda, a manobras inconsistentes como as tentadas pelos Bromberger. Eugen não esquecia, porém, que essas manobras racistas, por mais gratuitas que fossem, conseguiriam sempre assutar os mais timoratos. A situação de Lisbeth, face à chamada Nova Ordem Alemã, só não o procupava porque, sendo ela cidadã portuguesa, se encontrava a salvo de qualquer intromissão nazi, ainda que fosse através da via consular. Por simples questão de princípios éticos, porém, Eugen sentia-se sempre solidário com qualquer vítima de qualquer prepotência - e nunca, até aí, tivera a menor suspeita de que a vida fácil e agradável da colónia alemã residente no Funchal pudesse ocultar tensões e situações tão aberrantes. ["Tous ne disposaient pas, évidemment, comme le Docteur Franz Schönberg et lui-même, de la sécurité apportée par le fait de résider et de travailler à Madère depuis déjà plusieurs années, en complète légalité et parfaitement bien intégrés socialement - et tout aussi invulnérables, du fait de leur esprit et de leur tempérament, face à tout procédé intimidateur et encore moins face à des manœuvres inconsistantes comme celles que tentaient les Bromberger. Eugen n'oubliait pas, cependant, que ces manœuvres racistes avaient beau être gratuites, elles parviendraient toujours à effrayer les plus timorés. La seule raison pour laquelle la situation de Lisbeth, face au dit Nouvel Ordre Allemand ne le préoccupait pas, c'est parce qu'étant donné qu'elle était citoyenne portugaise, elle se trouvait protégée de toute intromission nazie, même si celle-ci se faisait par voie consulaire. Pour une simple question de principes éthiques, cependant, Eugen se sentait toujours solidaire de toute victime d'une quelconque prépotence - et jamais il n'avait soupçonné jusque-là que la vie facile et agréable de la colonie allemande résidant à Funchal pouvait occulter des tensions et situations si aberrantes"] (Marques 2010: 30-31)

Quand le prêtre anglican qui devait enfin les marier est lui aussi menacé et ne peut aller au bout de son engagement, Eugen et Lisbeth décident de s'unir malgré tout devant les seuls témoins dignes de leur confiance, leur famille et leurs amis, à défaut d'avoir pu le faire de manière officielle, bien décidés qu'ils sont à ne pas se faire voler une nouvelle fois leur union.

En arrière-plan de ces récits d'existence se dessinent les menaces qui pèsent progressivement sur l'Europe et les rumeurs et nouvelles qui se répandent, alors que les hommes sont 
"quase todos refugiados potenciais de uma guerra ainda por declarar" ["presque tous les réfugiés potentiels d'une guerre qui n'a toujours pas été déclarée"] (idem: 34). Dans la société plutôt aisée de Funchal, très marquée par l'influence de l'Angleterre, on reste prudent lors des soirées mondaines cosmopolites où nombre d'intellectuels portugais et étrangers se croisent - le roman se penchera particulièrement sur deux réfugiés, un peintre et un antiquaire. Tous sont attentifs à une "règle implicite":

[esta regra] estabelecia a necessidade vigilante de manter as conversas ligeiras e inócuas [mas] mantinham-se, inamovíveis, as preocupantes realidades político-militares europeias, cenário indesejável que todos temiam, mas reconheciam não poder alterar e, tacitamente, evitavam ["[cette règle] établissait la nécessité vigilante de faire en sorte que les conversations restent légères et bénignes" même si "continuaient à planer les inquiétantes réalités politico-militaires européennes, décor indésirable que tous redoutaient, mais qu'ils reconnaissaient ne pouvoir changer et qu'ils évitaient, tacitement"]. (Marques 2010: 22-23)

L'un des chapitres est particulièrement consacré à la formation de Miguel, jeune homme qui termine ses études de Sciences Politiques à Londres, et entretient une relation secrète avec Katherine, la gérante de l'hôtel de Funchal. Initié en politique par un camarade qui lui explique le massacre de Guernica et ce qui se trame en Europe, Miguel prendra conscience des enjeux européens qui se jouent alors que, de retour à Funchal, il remarquera l'apparente insouciance de la population: "o espectro de uma nova guerra não parece, na verdade, ensombrar ainda a vida quotidiana dos madeirenses. Os hotéis continuam cheios de estrangeiros das mais diversas nacionalidades (...)" ["le spectre d'une nouvelle guerre ne semble pas, en réalité, assombrir encore la vie quotidienne des habitants de Madère. Les hôtels continuent à s'emplir d'étrangers des plus diverses nationalités"] (idem: 84). Miguel travaillera pour le journal de Madère Notícias, et traduira les nouvelles transmises par l'agence Reuters arrivant de Londres par le télégraphe, donnant ainsi une voix à son engagement de citoyen éveillé aux dangers qui menacent le monde (idem: 183). Le sujet de la guerre imminente et de l'agissement des Allemands restera pourtant tabou avec sa petite amie, allemande (mais qui cache sa double nationalité suisse), dont il ne sait à quel bord elle appartient, d'autant plus que Katherine, de son côté, cache un autre secret - son affiliation au parti nazi, geste entrepris dans le seul but de se sauver et ne pas être inquiétée par les Bromberger, sur le conseil du consul allemand de Funchal (idem: 68), alors même qu'elle reçoit régulièrement dans l'hôtel des passagers allemands des bateaux de croisière qui font escale à Madère. Si aucune mention de la dictature portugaise n'est faite dans le roman, elle l'est cependant de manière implicite lorsqu'est évoqué l'un de ces voyages pensés par l'organisation nazie "Força pela Alegria" ("Force par la Joie" - "Kraft durch Freude" en allemand, plus communément appelée K.D.F), avec escale à Lisbonne, "capital de um país colaborante e amigo" ["capitale d'un pays collaborateur et ami"] (Marques 2010: 75). Cette organisation veillait à contrôler les loisirs des ouvriers allemands en leur proposant des croisières en Norvège, à Naples ou à Madère, à un prix défiant toute concurrence 
(Lauret 1945). Le débarquement de ces Allemands est le pendant terrible de celui des réfugiés qui ont fui le nazisme, dans un lieu qui symbolise, aussi bien pour les bourreaux que pour leurs victimes, un havre de paix et de beauté. Le roman met donc en scène le double jeu d'un pays et d'une société, à une époque où l'isolement et l'attractivité de Madère permettent de cultiver une apparente insouciance. Maria de Fátima Marinho analyse cette violence cachée derrière la façade idyllique, et note à ce sujet l'efficacité du titre du roman:

[o título] não deixa de aglutinar o núcleo essencial da trama e de significar a interferência dos valores alemães num espaço caracterizado pela amenidade e pelo bem-estar, lugar utópico, indiciador de uma mentalidade e de um estatuto social pré-definido, onde os estrangeiros se destacam por uma adaptação sem traumas ou discriminações.

[[ce titre] n'est pas sans concentrer l'essentiel de l'intrigue et de signifier l'interférence des valeurs allemandes dans un espace caractérisé par l'aménité et le bien-être, lieu utopique, indicateur d'une mentalité et d'un statut social prédéfini, où les étrangers se détachent par leur adaptation dénuée de traumas ou de discriminations]. (Marinho 2011: 237)

La psychologie des personnages, moins lisse qu'il n'y paraît parfois au premier abord, donne corps à cet esprit de suspicion - non seulement à la suspicion malsaine des Bromberger qui se croient tout permis et reproduisent le mécanisme de la délation, mais aussi à une suspicion généralisée: on soupçonne que la guerre va éclater sans oser la nommer, d'autres qu'on ne soupçonne pas cachent des secrets inavouables, d'autres encore sont instrumentalisés par des forces qui les dépassent et auxquelles ils sont forcés d'obéir, souvent malgré eux. Madère devient un décor en carton-pâte prêt à s'écrouler à tout moment, où coexistent l'être et le paraître, le dit et le non-dit, mais où s'exprime aussi à sa manière la résistance de ceux qui peuvent se permettre ce luxe. La stratégie narrative, centrée autour de trois procédés principaux, le narrateur omniscient à la troisième personne, les dialogues et les commentaires de la narratrice-autrice, comme l'analyse Maria de Fátima Marinho (ibidem) permet de donner de l'épaisseur aux personnages tout en confrontant le lecteur aux grands enjeux qui ont animé l'Europe et le monde dans les années 1930. Comme dans le documentaire de Nicholas Oulman, ces enjeux se confrontent à la coexistence de deux visions de la réalité, dans un Portugal aux deux visages. Si le documentaire insiste sur l'objectif commun de ces témoins (l'arrivée au Portugal), leur parcours de fuite encore enfants et les impressions qu'ils gardent de leurs sentiments d'alors, le roman d'Helena Marques concourt surtout à reproduire l'atmosphère d'une époque dans un noyau plus aisé de réfugiés ayant trouvé leur place au Portugal, dans une Europe prête à basculer. À l'innocence des enfants, transmise par les adultes qu'ils sont devenus, et que ces enjeux européens dépassaient à l'époque bien qu'ils en aient été des victimes, répond dans le roman d'Helena Marques le maintien voulu d'une certaine ignorance de ce que cache la réalité: on sait sans vouloir savoir, on voit sans vouloir voir, face à un mal qui agit parfois en sourdine. 
Ces deux œuvres nous livrent la mise à nu d'une époque et d'un espace présentés sous plusieurs facettes, parfois contradictoires mais toujours complémentaires. Le roman sur le passé de Madère nous fait entrer dans l'absurdité d'une mécanique de la délation, tandis que le documentaire de Nicholas Oulman vient combler les failles des images par les mots de ces autres témoins directs des événements. Le travail de création du langage et du montage vient ainsi enrichir une réalité plurielle dont nous nous devons de réactiver la mémoire.

\section{NOTES}

* Sandra Teixeira est Maître de Conférences à l'Université de Poitiers y enseigne la langue portugaise et la culture lusophone. Elle a publié de nombreux articles sur Vasco Graça Moura, auteur auquel elle a consacré sa thèse de doctorat soutenue en 2006 à l'Université de Paris 3-Sorbonne Nouvelle. Elle a par ailleurs publié des articles sur la littérature africaine de langue portugaise et a collaboré à quelques numéros de la revue portugaise Colóquio Letras. Elle a co-organisé le volume Donner un nom à l'obscur. Ecritures du divin, écritures du sacré dans la poésie ibérique et latino-américaine, Centre de Recherches Latino-Américaines - Archivos (Université de Poitiers - CNRS) / Amérique latine, Pays ibériques (Université Bordeaux Montaigne), 2015. Parmi ses dernières publications: "Mémoire intime et mémoire historique dans Que importa a fúria do mar, de Ana Margarida de Carvalho: l'archive vivante", Reflexos [En ligne], № 005, Engagement et désengagement dans les littératures et les arts des pays lusophones: l'archive, le manuscrit, mis à jour le : 30/05/2021, URL : http://revues.univ-tlse2.fr/reflexos/index. php?id=781 (14 pages); "Le Feu qui dort: um texto em chamas ou 'que farei quando tudo arde'”, in Basílio, Kelly Benoudis e Seixo, Maria Alzira (org.), Como uma Pedra no Silêncio. Recordar Mário Dionísio no Centenário do seu Nascimento, Lisboa, Univ. de Lisboa, Centro de Estudos Comparatistas, 2017, p. 178-181.

${ }^{1}$ Voir notamment le polar de Robert Wilson, Une mort à Lisbonne, 1999 (traduit en portugais sous le titre O Último acto em Lisboa, publié chez Gradiva en 2000 et chez D. Quixote en 2009). À partir du meurtre d'une jeune femme dans les années 1980 , le lecteur se voit plongé dans une saga familiale qui prend sa source dans la Seconde Guerre Mondiale et dans le trafic de wolfram (minerai également appelé "tungstène”), dont le Portugal était à l'époque l'un des principaux acteurs (cinquième pays producteur dans les années 1940), alimentant un vaste réseau clandestin avec les autorités nazies, alors que nombre de concessions étaient détenues par des capitaux anglais. Entre trafics et règlements de compte, transit des stocks d'or de la Suisse vers l'Espagne et le Portugal, puis vers le Brésil, c'est l'économie de toute une région (celle de la Beira, autour de la ville de Guarda), qui se retrouve bouleversée, le wolfram ayant attiré aussi bien les personnes les plus humbles que les cols blancs. (Voir à ce sujet le texte de Françoise Massa, "L'aventure du wolfram au Portugal", disponible sur <http://almalusa35.blogspot.com/search/label/Conf\%C3\%A9rence?updated-max=2016-11-05T15:40:00\%2B01:00\&max-results=20\&start=24\&by- 
date=false >). À travers le personnage de Miguel da Costa Rodrigues, le polar nous raconte l'ascension de son grand-père, Joaquim Abrantes, paysan ayant fait fortune grâce au trafic de wolfram et aux relations tissées avec l'Allemand Felsen, choisi par le régime nazi pour servir d'intermédiaire entre l'Allemagne et le Portugal. En arrière-plan sont évoqués les bombardements, les réfugiés et les massacres nazis, ainsi que les techniques employées par l'administration SS pour recruter des hommes de confiance capables de servir leurs intérêts.

${ }^{2}$ Il convient de préciser que cette étude de Georges Didi-Huberman porte sur l'observation phénoménologique de quatre photos prises à Auschwitz par un membre du Sonderkommando, ces prisonniers des camps chargés de manipuler les cadavres, et condamnés eux-mêmes à la mort (ils étaient régulièrement remplacés pour que ne fuite aucune donnée sur les procédés de gazage). Georges Didi-Huberman imagine dans quelles circonstances ont été prises ces photos, par des condamnés qui ont agi au péril de leur vie. Répondant, dans la deuxième partie du livre, à la polémique suscitée par son interprétation des images, qu'il défend au nom de l'impossibilité de fournir de cet événement historique des images qui reflètent "tout" l'événement, et de la nécessité de considérer ces bribes laissées par des témoins qui ont voulu sauver la mémoire de cet événement prétendument "inimaginable" et "indicible" (mais qu'on se doit, selon l'écrivain, d'imaginer et de dire, justement), Georges Didi-Huberman nous livre des analyses lumineuses sur le statut de l'image en général, et de l'archive en particulier. Les rapprochements qu'il effectue avec les écrits du réalisateur Jean-Luc Godard, notamment, sont tout à fait opérants pour une réflexion sur ces questions.

${ }^{3}$ Parmi la vingtaine de personnes entendues, Nicholas Oulman retient pour son film les témoignages d'Eva Arond, Lolita Goldstein, Fred Manasse, Pedro Kalb, Ginette Horowitz, Sylvain Bromberger et Henny Porter. La plupart vit aux Etats-Unis, et l'un d'eux en Argentine. Ils ont plus de quatre-vingts ans lorsqu'ils acceptent de rencontrer le réalisateur et de se replonger dans leurs souvenirs d'enfance. Trois de ces témoins étaient décédés depuis, au moment de la sortie du film (Sobral 2019; Pereira 2019).

4 Dans un entretien paru lors de la sortie du film, Nicholas Oulman évoque l'hospitalité que des Juifs ont trouvée chez sa famille installée à Dafundo. Il évoque également la manière dont les images de Berlin détruite semblent si familières et quotidiennes à notre époque, car elles font notamment écho aux images de la Syrie ravagée et aux flux de réfugiés que ces destructions ont provoqués. Voir <https://www.dn.pt/cultura/a-lisboa-de-salazar-foi-terreno-de-liberdade-para-quem-fugiu-ao-holocausto-10477039.html>, page consultée le 22 novembre 2020.

${ }^{5}$ Est évoquée "[a] imediata percepção de que ambos tinham já estabelecido uma sincera identificação com a terra que tão bem os acolhia, sentimento muito semelhante, por certo, ao que Franz lhe tinha manifestado: a convicção de ter chegado ao seu lugar certo no mundo, um lugar acolhedor e resguardado, precioso oásis numa Europa que se ia tornando, cada vez mais, ameaçadora e inquietante" [l'“immédiate perception que tous deux s'étaient déjà sincèrement identifiés à un pays qui les accueillait si bien, un sentiment sans doute très semblable à celui dont Franz lui avait fait part: la conviction d'être arrivé à l'endroit qui lui correspondait dans le monde, un endroit accueillant et protégé, précieuse oasis dans une Europe qui devenait de plus en plus menaçante et inquiétante"]. 
Bibliographie

Bensoussan, Georges (2015), "Éditorial", Revue d'Histoire de la Shoah, n²03, p. 7-19, <https://www.cairn.info/revue-revue-d-histoire-de-la-shoah-2015-2-page-7.htm> (page consultée le 13/07/20).

Carvalho, Miguel (2020), Amália. Ditadura e Revolução. A história secreta, Lisboa, Dom Quixote. Didi-Huberman, Georges (2003), Images malgré tout, Paris, Les Editions de Minuit.

Ketelaar, Éric (2006), “(Dé)construire l'archive", La contemporaine. Matériaux pour l'histoire de notre temps, $\mathrm{n}^{\circ} 82$, p. 65-70.

Lauret, René (1945), "Le Dr Ley, l'homme du KDF", Archives Le Monde, disponible sur <https://www.lemonde.fr/archives/article/1945/10/30/le-dr-ley-l-homme-du-kd-f_1856890_1819218.html> (page consultée le 10/08/21).

Marinho, Maria de Fátima (2011), recensão sobre Helena Marques, O Bazar alemão, Revista Colóquio/Letras. Recensões Críticas, n. ${ }^{0}$ 177, p. 236-239.

Marques, Helena (2010), o Bazar Alemão, Lisboa, Dom Quixote.

Meneses, Filipe Ribeiro de (2015), "Salazar face à la Shoah", traduit de l'anglais par Claire Darmon, Revue d'Histoire de la Shoah, n²03, p. 255-276, <https://www.cairn.info/revuerevue-d-histoire-de-la-shoah-2015-2-page-255.htm> (page consultée le 13/07/20).

Pereira, Mariana (2019), "A Lisboa de Salazar foi terreno de liberdade para quem fugiu ao Holocausto - sobre Debaixo do Céu, de Nicholas Oulman", Diário de Notícias, 24/01/2019 <https://www.dn.pt/cultura/a-lisboa-de-salazar-foi-terreno-de-liberdade-paraquem-fugiu-ao-holocausto-10477039.html> (page consultée le 22/11/ 2020).

Sobral Cláudia (2019), "Debaixo do Céu. O céu que é sempre o mesmo, para todos", Entrevista com Nicolas Oulman, Jornal ionline, <https://ionline.sapo.pt/artigo/643710/debaixodo-ceu-o-ceu-que-e-sempre-o-mesmo-para-todos?seccao=Mais $>$ (page consultée le $22 / 11 / 20$ ).

Ypsilantis, Olivier (2019), "Des mémoires juives et le refuge portugais, un film de Nicholas Oulman, 'Debaixo do Céu'", <https://zakhor-online.com/?p=15548> (page consultée le $22 / 11 / 20)$. 